\title{
Linear Pantographic Sheets: Existence and Uniqueness of Weak Solutions
}

\author{
Victor A. Eremeyev ${ }^{1,2,3}$ - Francesco dell'Isola ${ }^{3,4}$. \\ Claude Boutin ${ }^{5}$. David Steigmann 6
}

Received: 5 December 2016 / Published online: 6 November 2017

(C) The Author(s) 2017. This article is published with open access at Springerlink.com

\begin{abstract}
The well-posedness of the boundary value problems for second gradient elasticity has been studied under the assumption of strong ellipticity of the dependence on the second placement gradients (see, e.g., Chambon and Moullet in Comput. Methods Appl. Mech. Eng. 193:2771-2796, 2004 and Mareno and Healey in SIAM J. Math. Anal. 38:103-115, 2006).

The study of the equilibrium of planar pantographic lattices has been approached in two different ways: in dell'Isola et al. (Proc. R. Soc. Lond. Ser. A 472:20150, 2016) a discrete model was introduced involving extensional and rotational springs which is also valid in large deformations regimes while in Boutin et al. (Math. Mech. Complex Syst. 5:127-162, 2017) the lattice has been modelled as a set of beam elements interconnected by internal pivots, but the analysis was restricted to the linear case. In both papers a homogenized second gradient deformation energy, quadratic in the neighbourhood of non deformed configuration, is obtained via perturbative methods and the predictions obtained with the obtained
\end{abstract}

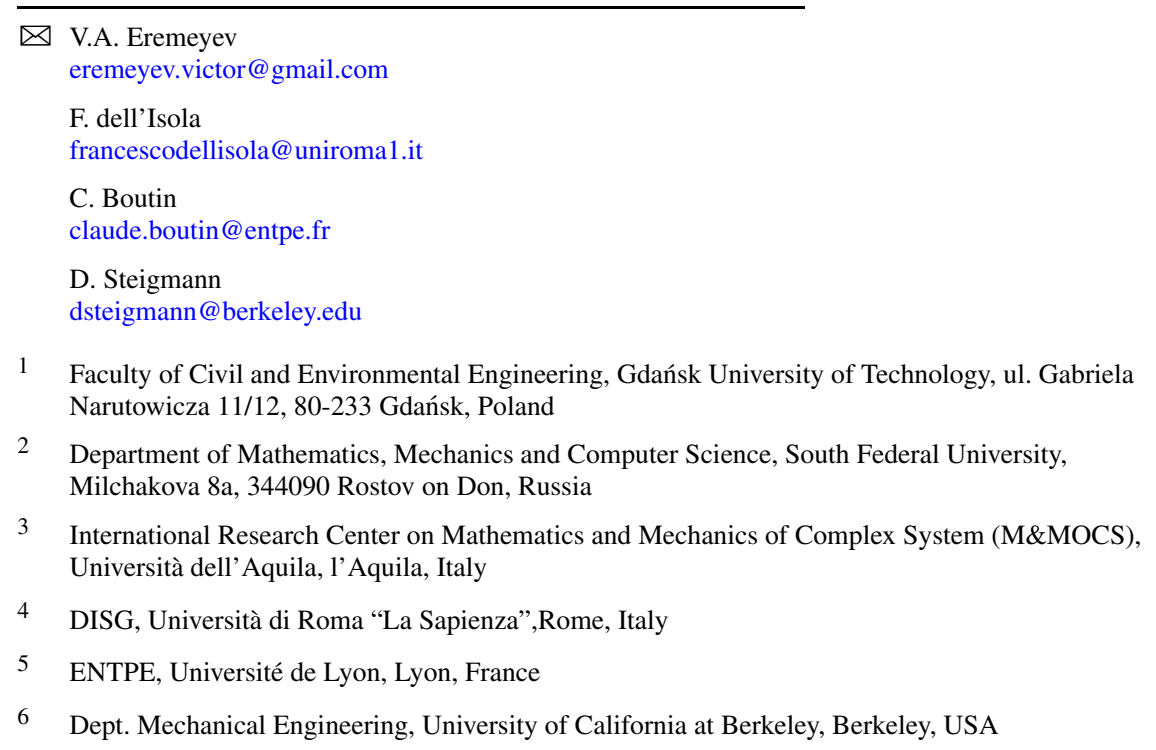


continuum model are successfully compared with experiments. This energy is not strongly elliptic in its dependence on second gradients. We consider in this paper also the important particular case of pantographic lattices whose first gradient energy does not depend on shear deformation: this could be considered either a pathological case or an important exceptional case (see Stillwell et al. in Am. Math. Mon. 105:850-858, 1998 and Turro in Angew. Chem., Int. Ed. Engl. 39:2255-2259, 2000). In both cases we believe that such a particular case deserves some attention because of what we can understand by studying it (see Dyson in Science 200:677-678, 1978). This circumstance motivates the present paper, where we address the well-posedness of the planar linearized equilibrium problem for homogenized pantographic lattices. To do so: (i) we introduce a class of subsets of anisotropic Sobolev's space as the most suitable energy space $E$ relative to assigned boundary conditions; (ii) we prove that the considered strain energy density is coercive and positive definite in $E$; (iii) we prove that the set of placements for which the strain energy is vanishing (the so-called floppy modes) must strictly include rigid motions; (iv) we determine the restrictions on displacement boundary conditions which assure existence and uniqueness of linear static problems. The presented results represent one of the first mechanical applications of the concept of Anisotropic Sobolev space, initially introduced only on the basis of purely abstract mathematical considerations.

Keywords Strain gradient elasticity · Linear pantographic sheets · Existence · Uniqueness · Anisotropic Sobolev's space

Mathematics Subject Classification 74A30 - 74G25 · 74G30 · 74G65 - 46E35

\section{Introduction}

Mechanical scientists have been recently attracted to the formulation of design and construction criteria of new materials whose behaviour is established $a$ priori. One can say that the aim of this stream of researches is to produce Materials on Demand. More precisely: once fixed the peculiar behaviour of a material which is desirable for optimizing its use in a given application, the aim of aforementioned researches is to find the way for constructing such a material. Materials designed in order to get a specific behaviour are often called metamaterials.

The role of mathematical sciences in the design and constructions of metamaterials recently increased for two reasons: (i) the development of the technology of 3D printing allowed for the transformation of the mathematically conceived structures, geometries and material properties into the reality of precisely built specimens; (ii) the way in which one specifies the set of properties to be realized is specifically mathematical, as this specification exactly consists in choosing the equations which one assumes to be those governing the mechanical response of the conceived metamaterial.

Once more we can say that mathematics is shaping our world, as it is allowing us to design new technological solutions and tools. The present paper deals with a mathematical problem arising in a specific context involving the design of second gradient metamaterials. More precisely: in order to find a class of materials whose deformation energy depends on both first and second gradient of placement field in [22] a microstructured (pantographic) fabric is introduced and its homogenized continuum model (which we call pantographic sheet) is determined. Various aspects of modelling of pantographic lattices are considered in $[8,52,53,56,62-66]$ where discrete and homogenized models are considered. Let us 
note that one of the sources of generalized continua and models of metamaterials is the homogenization of heterogeneous materials, see, e.g., $[18,54,61]$ and reference therein. Homogenization may lead also to strain gradient models $[14,17]$. While the ideas underlying the definition of pantographic microstructures have been exploited up to now only in the context of purely mechanical phenomena, it is expected that when introducing multi-physics effects (as the piezoelectric coupling phenomena exploited as explained in [23, 35]) the designed meta materials could have even more interesting features. We expect a fortiori that the mathematical tools used in the present context will be of use also in the envisioned more general context.

The linearized equilibrium equations valid in the neighbourhood of a stress free configuration for such pantographic sheets cannot be immediately studied by using the results available in the literature. However we prove in this paper that the standard strategy involving the use of Poincare inequality, Lax-Milgram Theorem and coercivity of bilinear deformation energy form do apply also in the present more generalized context.

What has to be modified is the Energy space where the solutions, relative to suitable well-posed boundary conditions, are looked for and the Sobolev space which includes this Energy Space.

Indeed the concept of Anisotropic Sobolev Space, whose definition was conceived on purely logical grounds by Sergei M. Nikol'skii, see [47], has to be used in order to apply the abstract Hilbertian setting of solution strategy.

We expect that further developments will lead us to study the complete nonlinear problem of deformation of pantographic sheets.

We need to explicitly remark here that we believe it is important to consider the particular case of pantographic lattices whose first gradient energy does not depend on shear deformation. This could be considered either a pathological case or an important exceptional object (in the sense made explicit in [58] and [67]). In both cases we believe that the pathology shown by pantographic lattices in absence of first gradient shear energy deserves due attention for what we can eventually understand by studying its ill-posedness or well-posedness. The significance of pathological examples in the development of mathematics has been widely discussed: without listing the many examples of pathological mathematical behaviour which has led to a better understanding of physical and mathematical world (we have found in the entry of Wikipedia (https://en.wikipedia.org/wiki/ Pathological_(mathematics) consulted on 17 April 2017, really interesting) we simply recall the classical example of the Greek discovery of irrational and its consequences (see, e.g., [38]). ${ }^{1}$

On the other hand if a behavior must be considered as pathological it is indeed subject to the personal intuition and therefore pathology is a concept which should not play a relevant role in scientific theories. What has to be classified as "pathology" depends on many factors including: its context, the training of the group of scientists studying the problem, and their experience: indeed what is regarded as pathological by one researcher may very well be

\footnotetext{
${ }^{1} \mathrm{Si}$ parva licet componere magnis (Virgil), i.e., if it be allowable to compare small things with great ones, we would like to cite in the present context a relevant statement by Freeman Dyson "The same pathological structures that the mathematicians invented to break loose from 19-th naturalism turn out to be inherent in familiar objects all around us in nature" in Characterizing Irregularity, Science 200 [1978] (see [25] for a deep and further discussion about this point). Moreover we believe to have pointed out in this paper an anomaly in classical elasticity theory (in the technical sense given to this word by Thomas Kuhn, see [39]). Indeed we prove here that the standard use of the hypothesis of ellipticity in the proof of well-posedness needs to be modified if one wants to incorporate linear second gradient elasticity in the body of theory of elasticity. The most conservative readers will be reassured by the fact that in this way it is avoided a crisis (always in the sense of Kuhn) inside this theory.
} 
standard behaviour to another one. The concept of pathologic behaviour seems of therefore of relevance only in the history of scientific thought and in the description of the process of solution finding.

\section{Postulated Deformation Energy for "Long-Fibers" Pantographic Sheets}

Pantographic sheets are bidimensional continua whose microstructure is constituted by a lattice of extensible and continuous fibers having bending stiffness and interconnected by (internal) pivots (i.e., pin joints which are not interrupting the material continuity of the pairs of beams to which they are applied). It has to be explicitly remarked that, in general, we are not considering trusses. A truss, by definition, is assumed to comprise a set of independent beams that are connected by means of pin joints connecting only ending points of the beams. This means that if the truss is loaded only with concentrated forces applied to pin joints then each beam (or fiber) can only be either in compression or in extension. We call lattices of beams the most general beams structure involving pin joints (but also possibly clamping devices, or rollers or glyphs).

Roughly speaking, pantographic sheets can be characterized as those lattices of fibers whose microstructure, once pivots are assumed to be ideal and no external constraints are applied, allows for the existence of some homogeneous deformations which do not store deformation energy. These deformations are sometimes called "floppy-modes". In Fig. 1 such a structure is schematically described, while in Fig. 2 a picture of a 3D printed specimen in polyamide is shown. Of course the pivots in the real object can have only an approximate ideal behaviour: they will store some deformation energy and therefore the homogenized corresponding continuum will need to have a non vanishing deformation energy relative to shear deformation. In our opinion, however, the ideal case of vanishing first gradient stiffness deserves a careful attention, as it can suggest to us some features and exotic behaviours which pantographic sheets can exhibit.

The main feature of the considered pantographic structure consists in the presence of "long" continuous fibers constituting two arrays: at each intersection point of one fiber with all fibers of the other array it is present a pin joint (or internal pivot) which is not interrupting the mechanical and geometrical continuity of both interconnecting fibers. This pin joint imposes that the beam sections which it is interconnecting must undergo the same displacement, however it leaves free their relative rotations.

We assume that in the reference configuration the two arrays of fibers are initially orthogonal and we denote $\mathbf{D}_{\alpha}, \alpha=1,2$, the unit vectors of their current directions.

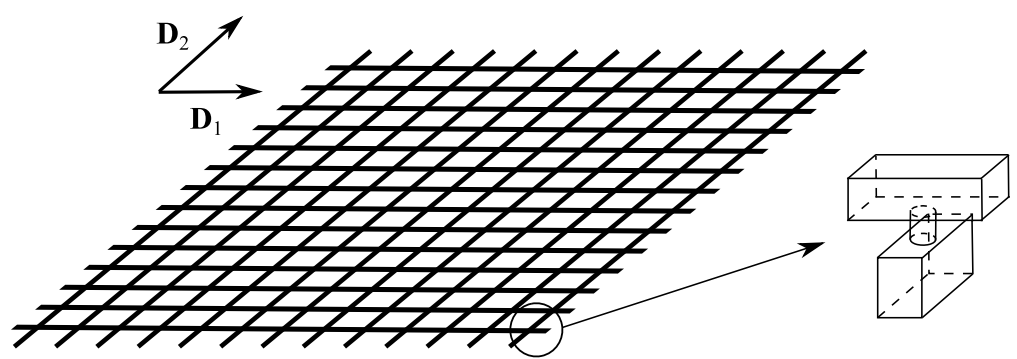

Fig. 1 Scheme of a pantographic sheet and beams connection trough a pivot 


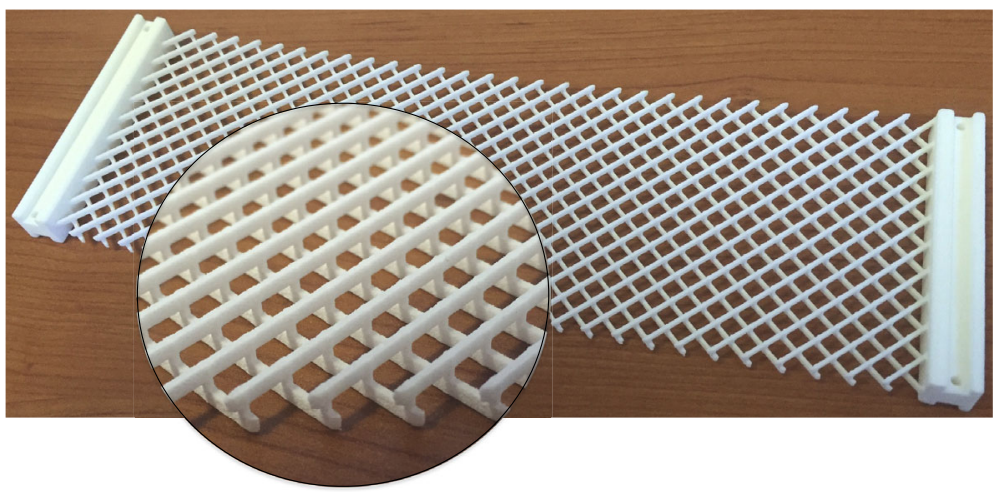

Fig. 2 3D printed specimen of a pantographic sheet

As a macro model of the system described before we consider a continuum whose reference configuration is given by a (suitably regular) domain $\omega \subset \mathbb{R}^{2}$. By assuming planar motion, the actual configuration of $\omega$ is described by the planar macro-placement

$$
\chi: \omega \rightarrow \mathbb{R}^{2},
$$

whose gradient $\nabla \chi$ will be denoted by $\mathbf{F}$.

For considered "long fibers" pantographic sheets a possible expression for deformation energy is given by (the two possible methods for getting this expression are described in [22] or in [12]):

$$
\begin{aligned}
U(\chi(\cdot))= & \iint_{\omega} \sum_{\alpha} \frac{\mathbb{K}_{e}^{\alpha}}{2}\left(\left\|\boldsymbol{F} \mathbf{D}_{\alpha}\right\|-1\right)^{2} d \omega \\
& +\iint_{\omega} \sum_{\alpha} \frac{\mathbb{K}_{b}^{\alpha}}{2}\left[\frac{\nabla \boldsymbol{F}: \mathbf{D}_{\alpha} \otimes \mathbf{D}_{\alpha} \cdot \nabla \boldsymbol{F}: \mathbf{D}_{\alpha} \otimes \mathbf{D}_{\alpha}}{\left\|\boldsymbol{F} \mathbf{D}_{\alpha}\right\|^{2}}\right. \\
& \left.-\left(\frac{\boldsymbol{F} \mathbf{D}_{\alpha}}{\left\|\boldsymbol{F} \mathbf{D}_{\alpha}\right\|} \cdot \frac{\nabla \boldsymbol{F}: \mathbf{D}_{\alpha} \otimes \mathbf{D}_{\alpha}}{\left\|\boldsymbol{F} \mathbf{D}_{\alpha}\right\|}\right)^{2}\right] d \omega \\
& +\iint_{\omega} \frac{\mathbb{K}_{p}}{2}\left|\arccos \left(\frac{\boldsymbol{F} \boldsymbol{D}_{1}}{\left\|\boldsymbol{F} \boldsymbol{D}_{1}\right\|} \cdot \frac{\boldsymbol{F} \boldsymbol{D}_{2}}{\left\|\boldsymbol{F} \boldsymbol{D}_{2}\right\|}\right)-\frac{\pi}{2}\right|^{2} d \omega,
\end{aligned}
$$

which accounts for stretching (first integral) and bending deformations of fibers (second integral) as well as for the resistance to shear distortion (third integral) related to the variation of the angle between the fibers. Here $\|\cdot\|$ is the Euclidean norm in $\mathbb{R}^{2}$ and : is the double dot product. The twisting deformations in fibers are not considering here. The coefficients $\mathbb{K}_{e}^{\alpha}>0$ and $\mathbb{K}_{b}^{\alpha}>0$ are related respectively to the extensional and bending stiffnesses of the fibers at the interpivot scale, while the coefficient $\mathbb{K}_{p} \geq 0$ models, at macro-level, the shear stiffness of the pantographic sheet and is related to the interaction between the two arrays of fibers via their interconnecting pivots: when these pivots are perfect this interaction is vanishing and $\mathbb{K}_{p}$ vanishes. Considering stiffness parameters $\mathbb{K}_{e}^{\alpha}$, $\mathbb{K}_{b}^{\alpha}$, and $\mathbb{K}_{p}$ we conclude that pivots are weakest elements in typical pantographic structures. An example of how printed fibers with pivots look like after breakage is given in Fig. 3. Even made of the same 


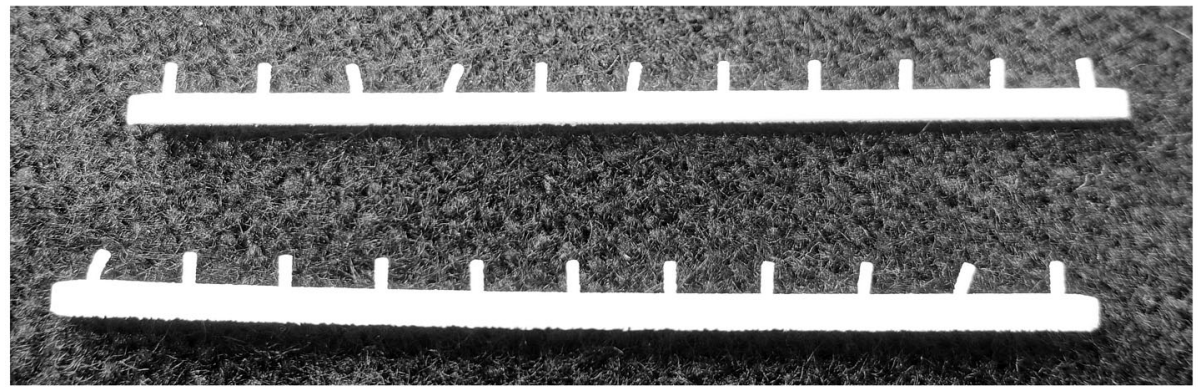

Fig. 3 Fibers with pivots

material, pivots are thinner than fibers. Thus, for such structures $\mathbb{K}_{p}$ is much smaller than the tangent and bending stiffnesses.

In this paper we will start considering small deformations of the sheet in the neighborhood of the reference configuration. Therefore we calculate the second order Taylor expansion for the energy $U(\chi(\cdot))$ in terms of the small parameter $\eta$ controlling the amplitude of the displacement $\mathbf{u}$ starting from the reference configuration. In formulas

$$
\chi(X)=\mathbf{X}+\eta \mathbf{u}(\mathbf{X}), \quad \mathbf{X} \in \omega .
$$

By introducing the notations $\mathbf{H}:=\nabla \mathbf{u}, \mathbf{H}=: \mathbf{E}+\mathbf{W}$, where $\mathbf{E}$ is symmetric and $\mathbf{W}$ is skew-symmetric we get formally (where $\mathbf{I}$ denotes the identity tensor)

$$
\mathbf{F}=\mathbf{I}+\eta \mathbf{H}, \quad \nabla \mathbf{F}=\eta \nabla \mathbf{H} .
$$

As a consequence

$$
\begin{aligned}
& \mathbf{F D}_{\alpha}=\mathbf{D}_{\alpha}+\eta \mathbf{H D}_{\alpha} \\
& \mathbf{F D}_{\alpha} \cdot \mathbf{F D}_{\alpha}=1+2 \eta \mathbf{H} \mathbf{D}_{\alpha} \cdot \mathbf{D}_{\alpha}+\eta^{2} \mathbf{H D}_{\alpha} \cdot \mathbf{H D}_{\alpha}, \\
& \left\|\mathbf{F D}_{\alpha}\right\|=\sqrt{1+2 \eta \mathbf{E D}_{\alpha} \cdot \mathbf{D}_{\alpha}+\eta^{2} \mathbf{H} \mathbf{D}_{\alpha} \cdot \mathbf{H D}_{\alpha}} \simeq 1+\eta \mathbf{H} \mathbf{D}_{\alpha} \cdot \mathbf{D}_{\alpha}, \\
& \frac{1}{\left\|\mathbf{F} \mathbf{D}_{\alpha}\right\|} \simeq 1-\eta \mathbf{H} \mathbf{D}_{\alpha} \cdot \mathbf{D}_{\alpha}, \\
& \frac{1}{\left\|\mathbf{F D}_{\alpha}\right\|^{2}} \simeq 1-2 \eta \mathbf{H} \mathbf{D}_{\alpha} \cdot \mathbf{D}_{\alpha} \\
& \frac{\mathbb{K}_{e}^{\alpha}}{2}\left(\left\|\mathbf{F D}_{\alpha}\right\|-1\right)^{2} \simeq \frac{\mathbb{K}_{e}^{\alpha}}{2}\left(\eta \mathbf{H} \mathbf{D}_{\alpha} \cdot \mathbf{D}_{\alpha}\right)^{2}, \\
& \frac{\boldsymbol{F} \boldsymbol{D}_{1}}{\left\|\boldsymbol{F} \boldsymbol{D}_{1}\right\|} \cdot \frac{\boldsymbol{F} \boldsymbol{D}_{2}}{\left\|\boldsymbol{F} \boldsymbol{D}_{2}\right\|} \simeq\left(\eta \mathbf{D}_{1} \cdot \mathbf{H D}_{2}+\eta \mathbf{H} \mathbf{D}_{1} \cdot \mathbf{D}_{2}\right) \\
& \frac{\mathbb{K}_{p}}{2}\left|\arccos \left(\frac{\boldsymbol{F} \boldsymbol{D}_{1}}{\left\|\boldsymbol{F} \boldsymbol{D}_{1}\right\|} \cdot \frac{\boldsymbol{F} \boldsymbol{D}_{2}}{\left\|\boldsymbol{F} \boldsymbol{D}_{2}\right\|}\right)-\frac{\pi}{2}\right|^{2} \simeq \frac{\mathbb{K}_{p}}{2}\left(\eta \mathbf{D}_{1} \cdot \mathbf{H D}_{2}+\eta \mathbf{H} \mathbf{D}_{1} \cdot \mathbf{D}_{2}\right)^{2}, \\
& \frac{\nabla \mathbf{F}: \mathbf{D}_{\alpha} \otimes \mathbf{D}_{\alpha} \cdot \nabla \mathbf{F}: \mathbf{D}_{\alpha} \otimes \mathbf{D}_{\alpha}}{\left\|\mathbf{F} \mathbf{D}_{\alpha}\right\|^{2}} \simeq \eta^{2}\left(\nabla \mathbf{H}: \mathbf{D}_{\alpha} \otimes \mathbf{D}_{\alpha} \cdot \nabla \mathbf{H} \mathbf{D}_{\alpha} \otimes \mathbf{D}_{\alpha}\right), \\
& \frac{\mathbf{F D}_{\alpha}}{\left\|\mathbf{F D}_{\alpha}\right\|} \cdot \frac{\nabla \mathbf{F} \mathbf{D}_{\alpha} \otimes \mathbf{D}_{\alpha}}{\left\|\mathbf{F D}_{\alpha}\right\|} \simeq\left(1-\eta \mathbf{H} \mathbf{D}_{\alpha} \cdot \mathbf{D}_{\alpha}\right)^{2}\left(\mathbf{D}_{\alpha}+\eta \mathbf{H} \mathbf{D}_{\alpha}\right) \cdot \eta \nabla \mathbf{H} \mathbf{D}_{\alpha} \otimes \mathbf{D}_{\alpha},
\end{aligned}
$$




$$
\frac{\mathbf{F D}_{\alpha}}{\left\|\mathbf{F D}_{\alpha}\right\|} \cdot \frac{\nabla \mathbf{F D}_{\alpha} \otimes \mathbf{D}_{\alpha}}{\left\|\mathbf{F D}_{\alpha}\right\|} \simeq \mathbf{D}_{\alpha} \cdot \eta \nabla \mathbf{H} \mathbf{D}_{\alpha} \otimes \mathbf{D}_{\alpha}
$$

Since $\mathbf{D}_{\alpha}$ are unit orthogonal vectors we may replace them by standard basis vectors $\mathbf{i}_{\alpha}$, $\mathbf{D}_{\alpha}=\mathbf{i}_{\alpha}$. As a consequence if the displacement $\mathbf{u}$ is represented in the basis $\mathbf{D}_{\alpha}$ by means of its components

$$
\mathbf{u}=u_{1} \mathbf{i}_{1}+u_{2} \mathbf{i}_{2}, \quad u_{\alpha}=u_{\alpha}\left(x_{1}, x_{2}\right), \quad \mathbf{X}=x_{1} \mathbf{i}_{1}+x_{2} \mathbf{i}_{2},
$$

then the second order Taylor expansion of the energy (2) is given by

$$
U(\mathbf{u}(\cdot))=\iint_{\omega}\left[\frac{\mathbb{K}_{e}^{1}}{2} u_{1,1}^{2}+\frac{\mathbb{K}_{e}^{2}}{2} u_{2,2}^{2}+\frac{\mathbb{K}_{p}}{2}\left(u_{1,2}+u_{2,1}\right)^{2}+\frac{\mathbb{K}_{b}^{1}}{2} u_{1,22}^{2}+\frac{\mathbb{K}_{b}^{2}}{2} u_{2,11}^{2}\right] d \omega,
$$

see also [12] for a direct derivation of this energy based on a homogenization procedure exploiting the micro-balance of force and momentum. In [51] the same energy has been derived with an explicit identification of the constitutive coefficients in terms of the Young's modulus of the fiber materials, of the area and of the moment of inertia of the fiber cross sections and of the distance between the nearest pivots. Here indices after comma denote derivatives, so $f_{, \alpha}$ is the partial derivative of $f$ with respect to $x_{\alpha}, f_{, \alpha}=\partial_{\alpha} f \equiv \frac{\partial f}{\partial x_{\alpha}}$.

Since the torsional (shear) stiffness is much smaller than other stiffness parameters used in the model we can study independently this singular limit case. So, in what follows and in the spirit described in the introduction, we mainly consider the mathematically interesting case which is represented by pantographic structures whose shear stiffness is vanishing.

\section{Energy for Pantographic Sheets and Equilibrium Conditions}

Let us consider the deformation energy relative to pantographic structures having vanishing shear stiffness. The deformation energy becomes

$$
U(\mathbf{u}(\cdot))=\iint_{\omega} W d \omega,
$$

where the strain energy density $W$ is given by

$$
W=\frac{\mathbb{K}_{e}^{1}}{2} u_{1,1}^{2}+\frac{\mathbb{K}_{e}^{2}}{2} u_{2,2}^{2}+\frac{\mathbb{K}_{b}^{1}}{2} u_{1,22}^{2}+\frac{\mathbb{K}_{b}^{2}}{2} u_{2,11}^{2} .
$$

For derivation of the equilibrium conditions we consider the first variation of $U$. First we obtain

$$
\delta U=\iint_{\omega}\left(\mathbb{K}_{e}^{1} u_{1,1} \delta u_{1,1}+\mathbb{K}_{e}^{2} u_{2,2} \delta u_{2,2}+\mathbb{K}_{b}^{1} u_{1,22} \delta u_{1,22}+\mathbb{K}_{b}^{2} u_{2,11} \delta u_{2,11}\right) d \omega .
$$

Then, integrating by parts we transform $\delta U$ into

$$
\begin{aligned}
\delta U= & \iint_{\omega}\left(-\mathbb{K}_{e}^{1} u_{1,11}+\mathbb{K}_{b}^{1} u_{1,2222}\right) \delta u_{1} d \omega \\
& +\iint_{\omega}\left(-\mathbb{K}_{e}^{2} u_{2,22}+\mathbb{K}_{b}^{2} u_{2,1111}\right) \delta u_{2} d \omega
\end{aligned}
$$




$$
\begin{aligned}
& +\int_{\partial \omega}\left[\left(n_{1} \mathbb{K}_{e}^{1} u_{1,1}-n_{2} \mathbb{K}_{b}^{1} u_{1,222}\right) \delta u_{1}+n_{2} \mathbb{K}_{b}^{1} u_{1,22} \delta u_{1,2}\right] d s \\
& +\int_{\partial \omega}\left[\left(n_{2} \mathbb{K}_{e}^{2} u_{2,2}-n_{1} \mathbb{K}_{b}^{2} u_{2,111}\right) \delta u_{2}+n_{1} \mathbb{K}_{b}^{2} u_{2,11} \delta u_{2,1}\right] d s .
\end{aligned}
$$

Here $n_{\alpha}=\mathbf{i}_{\alpha} \cdot \mathbf{n}, t_{\alpha}=\mathbf{i}_{\alpha} \cdot \mathbf{t}, \mathbf{n}$ and $\mathbf{t}$ are the unit external normal and unit tangent to the boundary $\partial \omega$ vectors, respectively, see Fig. 4 . Representing $\delta u_{1,2}$ and $\delta u_{2,1}$ by formulas

$$
\delta u_{1,2}=\mathbf{i}_{2} \cdot \nabla \delta u_{1}, \quad \delta u_{2,1}=\mathbf{i}_{1} \cdot \nabla \delta u_{2}
$$

with $\nabla$ defined at the boundary through normal and tangent derivatives

$$
\nabla=\mathbf{n} \frac{\partial}{\partial n}+\mathbf{t} \frac{\partial}{\partial s}
$$

where $\partial / \partial s$ and $\partial / \partial n$ are derivatives with respect to arc length $s$ and normal coordinate, respectively, we obtain that

$$
\delta u_{1,2}=n_{2} \frac{\partial \delta u_{1}}{\partial n}+t_{2} \frac{\partial \delta u_{1}}{\partial s}, \quad \delta u_{2,1}=n_{1} \frac{\partial \delta u_{2}}{\partial n}+t_{1} \frac{\partial \delta u_{2}}{\partial s} .
$$

Substituting the latter formulae into (6) and again integration by parts with respect to $s$ we obtain that

$$
\begin{aligned}
\delta U= & \iint_{\omega}\left[\left(-\mathbb{K}_{e}^{1} u_{1,11}+\mathbb{K}_{b}^{1} u_{1,2222}\right) \delta u_{1}+\left(-\mathbb{K}_{e}^{2} u_{2,22}+\mathbb{K}_{b}^{2} u_{2,1111}\right) \delta u_{2}\right] d \omega \\
& +\int_{\partial \omega}\left(n_{1} \mathbb{K}_{e}^{1} u_{1,1}-n_{2} \mathbb{K}_{b}^{1} u_{1,222}-\frac{\partial}{\partial s}\left(n_{2} t_{2} \mathbb{K}_{b}^{1} u_{1,22}\right)\right) \delta u_{1} d s \\
& +\int_{\partial \omega}\left(n_{2} \mathbb{K}_{e}^{2} u_{2,2}-n_{1} \mathbb{K}_{b}^{2} u_{2,111}-\frac{\partial}{\partial s}\left(n_{1} t_{1} \mathbb{K}_{b}^{2} u_{2,11}\right)\right) \delta u_{2} d s \\
& +\int_{\partial \omega}\left(\mathbb{K}_{b}^{1} u_{1,22} n_{2}^{2} \frac{\partial}{\partial n} \delta u_{1}+\mathbb{K}_{b}^{2} u_{2,11} n_{1}^{2} \frac{\partial}{\partial n} \delta u_{2}\right) d s .
\end{aligned}
$$

Fig. 4 Deformation of a pantographic sheet

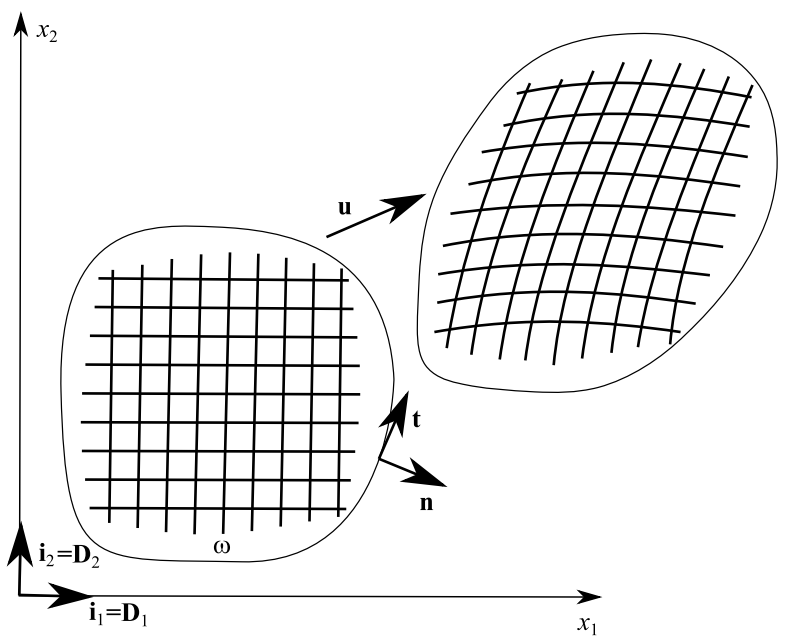


Here for simplicity we assumed that boundary contour $\partial \omega$ is a plane curve which is smooth enough, i.e., differentiable and without corner points. In $[50,51]$ the integration by part procedure valid for boundaries with corner points is considered. The form of $\delta U$ requires that only a class of external loads can be applied: indeed the virtual work of external loads must be consistent with it. So, we must assume that the virtual work of external loads $\delta A$ is given in the following form:

$$
\begin{aligned}
\delta A= & \iint_{\omega}\left(f_{1} \delta u_{1}+f_{2} \delta u_{2}\right) d \omega+\int_{\partial \omega}\left(\varphi_{1} \delta u_{1}+\varphi_{2} \delta u_{2}\right) d s \\
& +\int_{\partial \omega}\left(n_{2} \mu_{1} \frac{\partial}{\partial n} \delta u_{1}+n_{1} \mu_{2} \frac{\partial}{\partial n} \delta u_{2}\right) d s .
\end{aligned}
$$

Here $f_{\alpha}$ are surface loads. Moreover $\varphi_{\alpha}$ and $\mu_{\alpha}$ are forces and couples respectively assigned on the part of the boundary $\partial \omega$ where $u_{\alpha}$ and/or $\frac{\partial u_{\alpha}}{\partial n}$ are not assigned. Therefore we introduce a suitably regular partition of $\partial \omega$ into two disjoint subsets $\partial_{e} \omega_{\alpha}$ and $\partial_{n} \omega_{\alpha}$ (or $\partial_{e} \omega_{\alpha}^{\perp}$ and $\partial_{n} \omega_{\alpha}^{\perp}$ ) on which either displacements (or normal derivatives of displacements) are assigned or their dual quantities are assigned respectively (the index $\alpha=1,2$ refers to the displacement component $u_{\alpha}$ ).

Finally, from the principle of least action $\delta U-\delta A=0$, and by assuming the following essential boundary conditions,

$$
\begin{aligned}
u_{1} & =u_{1}^{0}, \quad\left(x_{1}, x_{2}\right) \in \partial_{e} \omega_{1}, \\
u_{2} & =u_{2}^{0}, \quad\left(x_{1}, x_{2}\right) \in \partial_{e} \omega_{2}, \\
n_{2} \partial_{n} u_{1} & =\vartheta_{1} n_{2}, \quad\left(x_{1}, x_{2}\right) \in \partial_{e} \omega_{1}^{\perp}, \\
n_{1} \partial_{n} u_{2} & =\vartheta_{2} n_{1}, \quad\left(x_{1}, x_{2}\right) \in \partial_{e} \omega_{2}^{\perp},
\end{aligned}
$$

where $u_{1}^{0}, u_{2}^{0}, \vartheta_{1}$, and $\vartheta_{2}$ are given functions at $\partial \omega$ and $\partial_{n}=\partial / \partial n$, we obtain the equilibrium equations and natural (static) boundary conditions

$$
\begin{gathered}
-\mathbb{K}_{e}^{1} u_{1,11}+\mathbb{K}_{b}^{1} u_{1,2222}-f_{1}=0, \quad\left(x_{1}, x_{2}\right) \in \omega, \\
-\mathbb{K}_{e}^{2} u_{2,22}+\mathbb{K}_{b}^{2} u_{2,1111}-f_{2}=0, \quad\left(x_{1}, x_{2}\right) \in \omega ; \\
n_{1} \mathbb{K}_{e}^{1} u_{1,1}-n_{2} \mathbb{K}_{b}^{1} u_{1,222}-\frac{\partial}{\partial s}\left(n_{2} t_{2} \mathbb{K}_{b}^{1} u_{1,22}\right)=\varphi_{1}, \quad\left(x_{1}, x_{2}\right) \in \partial_{n} \omega_{1} ; \\
n_{2} \mathbb{K}_{e}^{2} u_{2,2}-n_{1} \mathbb{K}_{b}^{2} u_{2,111}-\frac{\partial}{\partial s}\left(n_{1} t_{1} \mathbb{K}_{b}^{2} u_{2,11}\right)=\varphi_{2}, \quad\left(x_{1}, x_{2}\right) \in \partial_{n} \omega_{2} ; \\
\mathbb{K}_{b}^{1} u_{1,22} n_{2}^{2}=n_{2} \mu_{1}, \quad\left(x_{1}, x_{2}\right) \in \partial_{n} \omega_{1}^{\perp} ; \\
\mathbb{K}_{b}^{2} u_{2,11} n_{1}^{2}=n_{1} \mu_{2}, \quad\left(x_{1}, x_{2}\right) \in \partial_{n} \omega_{2}^{\perp} .
\end{gathered}
$$

It is interesting that (13) and (14) contain partial derivatives of different orders. For example, (13) contains second derivative with respect to $x_{1}$ and fourth derivative with respect to $x_{2}$.

Since the energy has a reduced form (in other words: in it some higher order derivatives are not appearing) we have also reduced boundary conditions. For example, for a fixed boundary and differently from what happens in the case of first gradient elasticity, instead of $\partial_{n} u_{1}=0$ we have $n_{2} \partial_{n} u_{1}=0$ which has a sense if $n_{2} \neq 0$. Indeed, if $n_{2}=0$ that corresponds 
Fig. 5 Pantographic rectangle

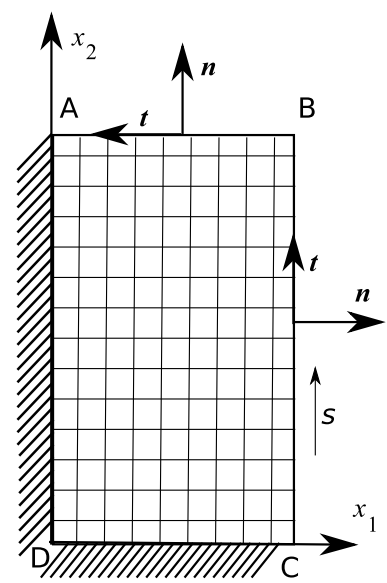

to a vertical line then the boundary condition (17) becomes trivial, both left and right parts of (17) vanish identically. Let us consider a rectangle $A B C D$ shown in Fig. 5. Note that here the fibers are oriented along the sides of the rectangle. Here two sides are free and at two sides the displacements are zero. The corresponding boundary conditions are

$$
\begin{array}{llll}
\text { along } A B: & \mathbb{K}_{b}^{1} u_{1,22}=0, & \mathbb{K}_{b}^{1} u_{1,222}=0, & \mathbb{K}_{e}^{2} u_{2,2}=0, \\
\text { along } B C: & \mathbb{K}_{e}^{1} u_{1,1}=0, & \mathbb{K}_{b}^{2} u_{2,111}=0, & \mathbb{K}_{b}^{2} u_{2,11}=0, \\
\text { along } C D: & u_{1}=0, & u_{2}=0, & \partial_{2} u_{1}=0,
\end{array}
$$

Clearly, this rectangle gives an example of degenerated boundary conditions, since instead of four conditions as in the general case, here we have only three. This circumstance occurs always when the boundary (or a part of it) is parallel to one of the coordinate axes, i.e., is parallel to one of the fiber directions.

\section{Heuristics}

It is evident that the immediate application of the classic methods used for proving existence and uniqueness of the solution of the elastic problem is not possible in the present context $[16,28,32,42]$, as coercivity could seem, at first sight, a condition which is not verified (this circumstance is already mentioned in [12]). Moreover also the results for second gradient continua proven by Healey et al. [36, 44] are not applicable here, as the energy of pantographic sheets is not coercive with respect to the highest order derivatives.

Before framing the problem in the appropriate energy space we present here some heuristic preliminary considerations.

First of all: assume that for a displacement field $\mathbf{u}^{*}$ the energy (5) vanishes.

It is trivial to check that as $u_{1,1}^{*}=0$

$$
u_{1}^{*}=f\left(x_{2}\right)
$$


while, as $u_{1,22}^{*}=f_{, 22}=0$

$$
f=a_{1} x_{2}+b_{1}
$$

and, finally,

$$
u_{1}^{*}=a_{1} x_{2}+b_{1},
$$

where $a_{1}$ and $b_{1}$ are constants. We have that

$$
u_{2}^{*}=a_{2} x_{1}+b_{2}
$$

with constant $a_{2}$ and $b_{2}$ independent of $a_{1}$ and $b_{1}$.

Note that in the case of plane infinitesimal deformations the rigid body motion is

$$
\mathbf{u}_{r}=\boldsymbol{\phi} \times \mathbf{X}+\mathbf{b},
$$

where $\phi=\phi \mathbf{i}_{3}$ is a constant rotation vector, $\mathbf{i}_{3}=\mathbf{i}_{1} \times \mathbf{i}_{2}, \times$ is the cross product, and $\mathbf{b}=$ $b_{1} \mathbf{i}_{1}+b_{2} \mathbf{i}_{2}$ is a constant vector. So in components the rigid body motion has the form

$$
u_{1}=\phi x_{2}+b_{1}, \quad u_{2}=-\phi x_{1}+b_{2} .
$$

It is therefore evident that the "kernel" or "null-space" of the strain energy not only include rigid (infinitesimal) motions (corresponding to $a_{1}=-a_{2}$ ) but also pure shear that corresponds to elongation/contraction in the directions at an angle $\pm \pi / 4$ with respect to the coordinate axes (when $a_{1}=a_{2}$ ). The null-space of strain energy density consists of four linear independent modes and all their linear combinations

$$
\mathbf{u}_{1}^{*}=\mathbf{i}_{1}, \quad \mathbf{u}_{2}^{*}=\mathbf{i}_{2}, \quad \mathbf{u}_{3}^{*}=\mathbf{i}_{3} \times \mathbf{X}, \quad \mathbf{u}_{4}^{*}=x_{2} \mathbf{i}_{1} .
$$

Instead of $\mathbf{u}_{4}^{*}$ one can use the equivalent mode $x_{1} \mathbf{i}_{2}$ or the symmetric mode $x_{2} \mathbf{i}_{1}+x_{1} \mathbf{i}_{2}$. Differently to what happens for first gradient elasticity a fourth mode having vanishing energy appears and it relates to uniform shear in a specific direction.

Clearly well-posedness results must take into account such a property.

Secondly: it has to be recalled that boundary conditions producing well-posed problems in the case of second gradient continua are more general than when dealing with first gradient continua (see for more details about generalized boundary conditions, e.g., [33, 34, 46, 59] and the historic works by Piola). The procedure which is used in the aforementioned papers can be summarized as follows (see [5, 19]): i) one postulates the principle of virtual work, i.e., the equality between internal and external work expended on virtual displacements; ii) one determines a class of internal work functionals involving second gradients of virtual displacement; iii) one determines, by means of an integration by parts, the class of external work functionals which are compatible with the determined class of internal work functionals.

A consequence (see, e.g., [24, 33, 46, 59]) of the just described procedure is that Neumann problems for considered second gradient deformation energies must include, to be complete, double symmetric and skew-symmetric boundary forces together with forces concentrated on points. To be more precise: the class of so-called natural boundary conditions must include the dual (with respect to work functionals) quantities of normal gradients of virtual displacements. Following Germain, the dual of the tangential part of normal gradient of virtual displacement is a "couple" (i.e., skew-symmetric contact double forces) while the dual of the normal part of normal gradient of virtual displacement is a "double force" (i.e., 
symmetric contact double forces). For some reasons (initially studied in [20, 21, 29], but surely further investigations are needed!) this kind of boundary conditions has been considered, sometimes and by some schools of mechanicians, unphysical: the reader is referred to the beautiful paper by Sedov, Leonid Ivanovich, [57] for a lucid discussion of this point and its physical, mathematical and epistemological implications.

After having identified the displacements which are in the null space of the deformation energy, a conjecture about mixed boundary conditions which are likely to produce wellposed problems can, consequently, be formulated. Indeed, let us partition the boundary $\partial \omega$ of the body $\omega$ into two disjoint subsets, i.e., $\partial \omega_{e}$ and $\partial \omega_{n}$. We assume that the displacements on $\partial \omega_{e}$ are assigned and that the displacements on $\partial \omega_{n}$ are free. We call $A C$ the set of $C^{2}$ displacements verifying the assigned conditions on $\partial \omega_{e}$. We say that $A C$ is singular if there exist an element $\mathbf{u}$ in $A C$ and a displacement field $\mathbf{u}_{0}$ belonging to the null space for deformation energy (i.e., a displacement having vanishing deformation energy) such that $\mathbf{u}+\mathbf{u}_{0}$ also belongs to $A C$. We conjecture here (and rigorously prove in the next section) that the considered mixed boundary problem is well-posed if and only if $A C$ is NOT singular. Remark that if the considered elastic energy is a first gradient one and it is positive when regarded as a function of infinitesimal strain tensor then the aforementioned statement reduces to the standard requirement that in well-posed problems the constrained body cannot undergo rigid displacements. The concept of underconstrained system (see [40]) has to be modified in order to include the treated case of planar second gradient continua: see, for instance, Figs. 1 and 2 for examples of underconstrained pantographic sheets. This point will need further investigations to include the case of pantographic sheets moving in threedimensional space and three-dimensional pantographic bodies.

In the present paper we limit ourselves to consider Dirichlet's and mixed boundary problems in which, on a part of the body boundary, only the displacement is assigned, while the remaining part of the body boundary is left free.

\section{On Ellipticity of Equilibrium Equations}

The equilibrium equations (13) and (14) are very specific. Indeed, unlike classic mechanical models like membranes, plates, or linear elastic solids they are neither elliptic nor strongly elliptic. Let us introduce the differential operators

$$
P_{1}(D)=-\mathbb{K}_{e}^{1} \partial_{1}^{2}+\mathbb{K}_{b}^{1} \partial_{2}^{4}, \quad P_{2}(D)=-\mathbb{K}_{e}^{2} \partial_{2}^{2}+\mathbb{K}_{b}^{2} \partial_{1}^{4}, \quad D=\left(\partial_{1}, \partial_{2}\right) .
$$

So (13) and (14) take a symbolic form

$$
P_{1}(D) u_{1}=f_{1}, \quad P_{2}(D) u_{2}=f_{2} .
$$

Then the ellipticity of $P_{1}(D)$ and $P_{2}(D)$ requires that, see [31, 32],

$$
\mathbb{K}_{b}^{1} \xi_{2}^{4} \neq 0, \quad \mathbb{K}_{b}^{2} \xi_{1}^{4} \neq 0, \quad \forall \xi \equiv\left(\xi_{1}, \xi_{2}\right) \neq \mathbf{0},
$$

respectively. Obviously, these conditions are not fulfilled.

Strong ellipticity requires the fulfillment of a stronger condition such as

$$
\mathbb{K}_{b}^{1} \xi_{2}^{4} \geq c_{1}|\boldsymbol{\xi}|^{4}, \quad \mathbb{K}_{b}^{2} \xi_{1}^{4} \geq c_{2}|\boldsymbol{\xi}|^{4}, \quad \forall \boldsymbol{\xi} \equiv\left(\xi_{1}, \xi_{2}\right) \neq \mathbf{0}
$$

with positive constants $c_{1}$ and $c_{2}$, see [4] for the general framework, and [44] for the strain gradient elasticity. So, it is clear that here the equilibrium equations are not strongly elliptic. 
Nevertheless, the considered operators are still elliptic in the sense of Agmon-DouglisNirenberg $[2,3]$. The differential operators $P_{1}(D)$ and $P_{2}(D)$ belong to the class of quasielliptic or semi-elliptic differential operators. The boundary value problems for quasi-elliptic operators have been intensively studied at the end of the Sixties, see, e.g., [6, 7, 13, 45, 68]. The quasi-elliptic operators are a particular class of the so-called hypoelliptic operators, see, e.g., [37]. It is easy to prove that $P_{1}(D)$ and $P_{2}(D)$ are hypoelliptic. Indeed, necessary and sufficient condition for the hypoellipticity of the operator $P(D)$ is the following limiting relation $[27,37]$

$$
\frac{|\nabla P(\xi)|}{|P(\xi)|} \rightarrow 0, \quad|\xi| \rightarrow+\infty,
$$

where $\nabla P=\left(\frac{\partial P}{\partial \xi_{1}}, \frac{\partial P}{\partial \xi_{2}}\right)$, which is fulfilled for $P_{1}(D)$ and $P_{2}(D)$.

For the theory of elliptic, quasi-elliptic and hypoelliptic operators we refer to $[4,27,37$, $43,49,55]$ and the references therein. In particular, in [43, p. 214] the variational statement of similar problem for an rectangle is discussed.

\section{Existence and Uniqueness of Weak Solutions}

Let us now come back to the first variation of the energy functional. For solution we have the principle of virtual work in the form

$$
\delta U-\delta A=0 .
$$

For simplicity let us replace $\delta \mathbf{u}$ by a new function $\mathbf{v}$. With $\mathbf{v}$ Eq. (21) transforms to

$$
\begin{aligned}
B(\mathbf{u}, \mathbf{v}) \equiv & \iint_{\omega}\left(\mathbb{K}_{e}^{1} u_{1,1} v_{1,1}+\mathbb{K}_{e}^{2} u_{2,2} v_{2,2}+\mathbb{K}_{b}^{1} u_{1,22} v_{1,22}+\mathbb{K}_{b}^{2} u_{2,11} v_{2,11}\right) d \omega \\
= & \iint_{\omega}\left(f_{1} v_{1}+f_{2} v_{2}\right) d \omega \\
& +\int_{\partial \omega}\left(\varphi_{1} v_{1}+\varphi_{2} v_{2}+n_{2} \mu_{1} \frac{\partial v_{1}}{\partial n}+n_{1} \mu_{2} \frac{\partial v_{2}}{\partial n}\right) d s
\end{aligned}
$$

Here we introduce the bilinear form $B(\mathbf{u}, \mathbf{v})$ to denote the quadratic terms in (21).

Now we introduce the weak solution of the boundary-value problem (13)-(17) as the vector-function $\mathbf{u}$ such that the variational equation (22) is fulfilled for any test function $\mathbf{v}=v_{1} \mathbf{i}_{1}+v_{2} \mathbf{i}_{2}$. The properties of $\mathbf{u}$ and $\mathbf{v}$ are specialized below.

Without loss of generality, in what follows we use the following dimensionless form of $W:$

$$
2 W=u_{1,1}^{2}+u_{2,2}^{2}+u_{1,22}^{2}+u_{2,11}^{2} .
$$

Now the bilinear form is

$$
B(\mathbf{u}, \mathbf{v})=\iint_{\omega}\left(u_{1,1} v_{1,1}+u_{2,2} v_{2,2}+u_{1,22} v_{1,22}+u_{2,11} v_{2,11}\right) d \omega
$$

Here we keep the same notations $u_{\alpha}$ and $x_{\alpha}$ for dimensionless displacements and dimensionless coordinates, respectively. So, $W$ has the form of a seminorm in anisotropic Sobolev 
spaces, see definition in [9-11], and Appendix. More precisely: let $u_{1} \in W_{2}^{(1,2)}(\omega)$ and $u_{2} \in W_{2}^{(2,1)}(\omega)$, then we have that $\mathbf{u} \in W_{2}^{(1,2)}(\omega) \oplus W_{2}^{(2,1)}(\omega)$ and

$$
2 U(\mathbf{u})=\iint_{\omega}\left[u_{1,1}^{2}+u_{2,2}^{2}+u_{1,22}^{2}+u_{2,11}^{2}\right] d \omega=\left|u_{1}\right|_{W_{2}^{(1,2)}}+\left|u_{2}\right|_{W_{2}^{(2,1)}}
$$

is a seminorm in $W_{2}^{(1,2)}(\omega) \oplus W_{2}^{(2,1)}(\omega)$. Here we introduced the following notations for some auxiliary seminorms

$$
|f|_{W_{2}^{(1,2)}}:=\left\|f_{, 1}\right\|_{L_{2}}+\left\|f_{, 22}\right\|_{L_{2}}, \quad|f|_{W_{2}^{(2,1)}}:=\left\|f_{, 11}\right\|_{L_{2}}+\left\|f_{, 2}\right\|_{L_{2}},
$$

whereas it is possible to transform them into norms for instance following the standard choice:

$$
\|f\|_{W_{2}^{(1,2)}}=\|f\|_{L_{2}}+|f|_{W_{2}^{(1,2)}}, \quad\|f\|_{W_{2}^{(2,1)}}=\|f\|_{L_{2}}+|f|_{W_{2}^{(2,1)}} .
$$

It clear that the functional space $W_{2}^{(1,2)}(\omega) \oplus W_{2}^{(2,1)}(\omega)$ is constituted exactly by the set of all functions for which (24) is finite. We will call energy space $E$ for the considered energy functional any subspace of $W_{2}^{(1,2)}(\omega) \oplus W_{2}^{(2,1)}(\omega)$ which is the completion of one of the previously introduced space $A C$ relative to NONSINGULAR boundary conditions using the norms (25). Remark that when restricted to an energy space the seminorm given by (24) becomes a norm.

Now the definition of a weak solution for linear pantographic structures can be given as follows.

Definition 1 We call $\mathbf{u} \in E$ a weak solution of the equilibrium problem (21) if (22) is fulfilled for any test function $\mathbf{v}$ from a dense set in $E$.

The bilinear form $B(\mathbf{u}, \mathbf{v})$ is continuous and the following inequalities are valid:

$$
B(\mathbf{u}, \mathbf{v}) \leq\left|u_{1}\right|_{W_{2}^{(1,2)}}\left|v_{1}\right|_{W_{2}^{(1,2)}}+\left|u_{2}\right|_{W_{2}^{(2,1)}}\left|v_{2}\right|_{W_{2}^{(2,1)}} \leq\|\mathbf{u}\|_{E}\|\mathbf{v}\|_{E} .
$$

For the analysis of existence and uniqueness of weak solutions we start by considering two cases. The simplest case is given by Dirichlet boundary conditions.

\subsection{Dirichlet's Boundary Conditions}

We start by proving the existence and uniqueness for the simplest case when the whole boundary is fixed. Consider the set of equations for the strong formulation of equilibrium problem:

$$
\begin{gathered}
\quad-u_{1,11}+u_{1,2222}=f_{1}, \quad-u_{2,22}+u_{2,1111}=f_{2}, \quad\left(x_{1}, x_{2}\right) \in \omega ; \\
u_{1}=0, \quad u_{2}=0, \quad n_{2} \partial_{n} u_{1}=0, \quad n_{1} \partial_{n} u_{2}=0, \quad\left(x_{1}, x_{2}\right) \in \partial \omega .
\end{gathered}
$$

Here the weak solution is defined through the integral equation

$$
B(\mathbf{u}, \mathbf{v})=\iint_{\omega}\left(f_{1} v_{1}+f_{2} v_{2}\right) d \omega, \quad \forall v_{1}, v_{2} \in C_{0}^{2}(\omega),
$$


which, when assuming that $\mathbf{f}$ belongs to $L_{2}(\omega)$, can be written as

$$
B(\mathbf{u}, \mathbf{v})-(\mathbf{f}, \mathbf{v})_{L_{2}}=0, \quad \forall \mathbf{v} \in C_{0}^{2}(\omega) .
$$

Using the Poincaré inequalities (Friedrich's inequality, see, e.g., [1]) we get

$$
\left\|u_{1}\right\|_{L_{2}} \leq C_{1}\left\|u_{1,1}\right\|_{L_{2}}, \quad\left\|u_{2}\right\|_{L_{2}} \leq C_{1}\left\|u_{2,2}\right\|_{L_{2}}
$$

with some constants $C_{1}$ and $C_{2}$ and, as a consequence, we can establish that

$$
\left|u_{1}\right|_{W_{2}^{(1,2)}}+\left|u_{2}\right|_{W_{2}^{(2,1)}} \geq C\left(\left\|u_{1}\right\|_{W_{2}^{(1,2)}}+\left\|u_{2}\right\|_{W_{2}^{(2,1)}}\right)
$$

with another constant $C$. In other words we proved that $|\cdot|_{W_{2}^{(1,2)}}$ and $|\cdot|_{W_{2}^{(2,1)}}$ play the role of norms in $\stackrel{\circ}{W}_{2}^{(1,2)}$ and $\stackrel{\circ}{W}_{2}^{(2,1)}$, respectively, where with the upper circle we denoted the completion of $C_{0}^{2}(\omega)$ (or $C_{0}^{\infty}(\omega)$ ) with respect to the corresponding norms. So here the energy space $E$ is the anisotropic Sobolev's space $\stackrel{\circ}{W}_{2}^{(1,2)}(\omega) \oplus \stackrel{\circ}{W} \stackrel{(2,1)}{2}(\omega)$. This means that we have proven that $B(\mathbf{u}, \mathbf{v})$ is coercive

$$
B(\mathbf{u}, \mathbf{u}) \geq C\|\mathbf{u}\|_{E}^{2} .
$$

One can easily prove that $(\mathbf{f}, \mathbf{v})_{L_{2}}$ is a linear bounded functional in $E$. Thus, by using Lax-Milgram theorem [30], the following theorem can be easily proven:

Theorem 1 Let the Cartesian components $f_{1}$ and $f_{2}$ of $\mathbf{f}$ belong to the space $L_{2}(\omega)$. There exists a weak solution $\mathbf{u}^{*} \in E \equiv \stackrel{\circ}{W}_{2}^{(1,2)}(\omega) \oplus \stackrel{\circ}{W}_{2}^{(2,1)}(\omega)$ to the equilibrium problem (27) and (28), which for any $\mathbf{v} \in E$ satisfies the equation

$$
\left(\mathbf{u}^{*}, \mathbf{v}\right)_{E}-\iint_{\omega} \mathbf{f} \cdot \mathbf{v} d \omega=0
$$

Furthermore, $\mathbf{u}^{*}$ is unique and it is a minimizer of the energy:

$$
F\left(\mathbf{u}^{*}\right)=\inf _{\mathbf{u} \in E} F(\mathbf{u}), \quad F(\mathbf{u}) \equiv U(\mathbf{u})-\iint_{\omega} \mathbf{f} \cdot \mathbf{u} d \omega .
$$

Remark 1 Since for the coercivity we need inequalities (30) which require that only the functions are zero at the boundary, (i.e., $u_{1}=u_{2}=0$ at $\partial \omega$ ), for uniqueness is suffices to consider only the boundary conditions concerning displacements, without considering the condition on the normal derivatives (28).

Remark 2 We used here $L_{2}(\omega)$ as a functional space for $\mathbf{f}$. This condition can be weakened using imbedding theorems of $E$ into anisotropic Lebesgue spaces [9-11] and we omit this for simplicity.

For non-homogeneous boundary conditions (9)-(12) we seek a solution in the form $\mathbf{u}=\mathbf{u}^{*}+\mathbf{u}_{0}$, where $\mathbf{u}_{0}$ is a vector function which satisfies (9)-(12) whereas for $\mathbf{u}^{*}$ boundary conditions (28) are assumed. Substituting this representation into (27) and (9)-(12) we reduce the non homogeneous boundary-value problem to the previous one, for which we already proved the theorem on existence and uniqueness of weak solutions. 
Fig. 6 Two rectangles with clamped edge with different fiber orientation

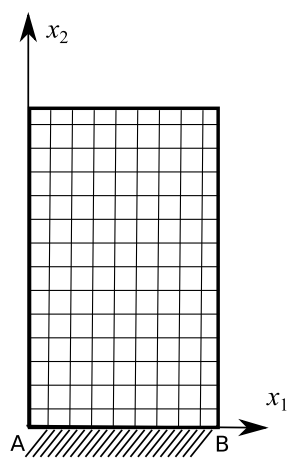

a)

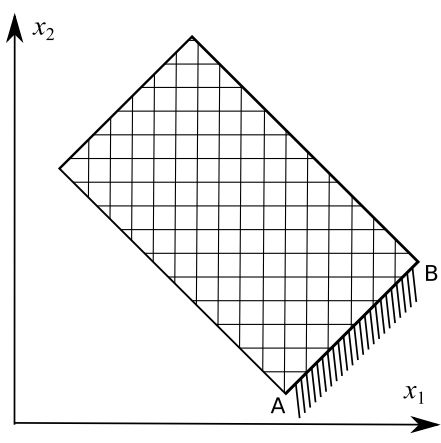

b)

\subsection{Mixed Boundary Conditions}

Somehow more difficult is the case of mixed boundary conditions. In the theory of linear elasticity it is known that for existence and uniqueness it is enough to request that a part of the boundary is fixed: i.e., when rigid displacements are not allowed [16, 28, 32, 42]. In our problem it is not enough to require that rigid displacements are ruled out. Indeed as an example we consider two rectangles with one fixed side whereas others are free, see Fig. 6. More precisely, on side $A B$ the displacements are zero: $\mathbf{u}=\mathbf{0}$. The difference in rectangles consists only on their orientation with respect to the coordinate axes, that is to fiber orientations. It is clear that for the first rectangle the solution is not unique since the displacement

$$
\mathbf{u}=a x_{2} \mathbf{i}_{1}
$$

satisfies the equilibrium and boundary conditions for any value of $a$ : the set $A C$ in this circumstance is indeed SINGULAR. For the second rectangle, instead, the set $A C$ is actually NON-SINGULAR and the aforementioned displacement is not a solution of equilibrium problem.

In other words, for rectangle a) we have at least the solutions $\mathbf{u}=\mathbf{0}$ and $\mathbf{u}=a x_{2} \mathbf{i}_{1}$. Obviously we should avoid such situations since even without loading we have an infinity of non-trivial (deformative) solutions. Thus, in what follows we always assume that the boundary conditions are nonsingular.

Let us consider the mixed boundary-value problem formulated by (9)-(18). Here the energy space $E$ is a subspace of $W_{2}^{(1,2)}(\omega) \oplus W_{2}^{(2,1)}(\omega)$ obtained as the completion of functions $C^{2}(\omega)$ which verify (9)-(12).

The weak solution is defined as an element $\mathbf{u}$ belonging to $E$ satisfying the equation

$$
\begin{aligned}
B(\mathbf{u}, \mathbf{v})= & \iint_{\omega}\left(f_{1} v_{1}+f_{2} v_{2}\right) d \omega \\
& +\int_{\partial \omega}\left(\varphi_{1} v_{1}+\varphi_{2} v_{2}+n_{2} \mu_{1} \frac{\partial v_{1}}{\partial n}+n_{1} \mu_{2} \frac{\partial v_{2}}{\partial n}\right) d s
\end{aligned}
$$

for any admissible function $\mathbf{v}$ (i.e., a function belonging to a dense subset of $E$ ).

Using the same technique we formulate the theorem on existence and uniqueness of the weak solution in $E$. 
Theorem 2 Let the Cartesian components $f_{1}$ and $f_{2}$ of $\mathbf{f}$ belong to the space $L_{2}(\omega), \varphi_{\alpha} \in$ $L_{2}\left(\partial_{n} \omega_{\alpha}\right), \mu_{\alpha} \in L_{2}\left(\partial_{n} \omega_{\alpha}^{\perp}\right)$ and assume that the boundary conditions are nonsingular. There exists a weak solution $\mathbf{u}^{*} \in E$ to the equilibrium problem (12)-(18) which for any $\mathbf{v} \in E$ satisfies the equation (31).

Furthermore, $\mathbf{u}^{*}$ is unique and it is a minimizer of the functional $F(\mathbf{u})$ :

$$
\begin{gathered}
F\left(\mathbf{u}^{*}\right)=\inf _{\mathbf{u} \in E} F(\mathbf{u}), \\
F(\mathbf{u}) \equiv U(\mathbf{u})-\iint_{\omega} \mathbf{f} \cdot \mathbf{u} d \omega+\int_{\partial \omega_{1}}\left(\varphi_{1} u_{1}+\varphi_{2} u_{2}+n_{2} \mu_{1} \frac{\partial u_{1}}{\partial n}+n_{1} \mu_{2} \frac{\partial u_{2}}{\partial n}\right) d s .
\end{gathered}
$$

\subsection{Existence and Uniqueness Considering Non-zero Shear Stiffness}

Using the anisotropic Sobolev spaces we can also prove existence and uniqueness for a more general case by considering energy in the form of (4). In dimensionless form the energy is given by

$$
2 U(\mathbf{u})=\iint_{\omega}\left[u_{1,1}^{2}+u_{2,2}^{2}+\left(u_{1,2}+u_{2,1}\right)^{2}+u_{1,22}^{2}+u_{2,11}^{2}\right] d \omega,
$$

and the bilinear form becomes

$$
\begin{aligned}
B(\mathbf{u}, \mathbf{v})= & \iint_{\omega}\left[u_{1,1} v_{1,1}+u_{2,2} v_{2,2}+\left(u_{1,2}+u_{2,1}\right)\left(v_{1,2}+v_{2,1}\right)\right. \\
& \left.+u_{1,22} v_{1,22}+u_{2,11} v_{2,11}\right] d \omega .
\end{aligned}
$$

Differently from the previous case where the energy is exactly a seminorm in $W_{2}^{(1,2)}(\omega) \oplus$ $W_{2}^{(2,1)}(\omega)$, now $U$ is slightly different. Moreover, for the energy (32) there are only rigid body motions as in the case of the classical plane elasticity. Considering Dirichlet or mixed boundary conditions with $u_{1}=0, u_{2}=0$ at the part of the boundary we can use the Korn inequality $[16,32,42]$ to prove the coercivity condition

$$
B(\mathbf{u}, \mathbf{u}) \geq C\|\mathbf{u}\|_{E}^{2} .
$$

Here the energy space $E$ is a subspace of $W_{2}^{(1,2)}(\omega) \oplus W_{2}^{(2,1)}(\omega)$ obtained as the completion of functions $C^{2}(\omega)$ which verify kinematic boundary conditions in the norm given by (32). Using Lax-Milgram theorem we can prove existence and uniqueness of the weak solutions. Let us note that this problem is much easier for analysis than the problem with zero shear stiffness. However the results given in the previous section gives us (i) the logical basis needed for formulating a numerical integration scheme which can be used in the case of small shear stiffness and (ii) a basis for understanding the exotic behaviour of purely second gradient pantographic sheets.

\section{Conclusions}

The results presented in this paper allow us to prove existence and uniqueness theorems for the elastic problem in the case of planar pantographic sheets and for a variety of boundary conditions. The main difficulties which we had to confront were: i) the existence of floppy modes, i.e., deformations corresponding to zero deformation energy and ii) the absence in the deformation energies of many higher order derivatives. 
Therefore the results by Healey and Chambon [15, 36, 44] could not be applied directly and there was the appearance of a lack of the coercivity of considered energy. Indeed the second gradient deformation energy for pantographic sheets is not coercive if one considers the standard Sobolev Space, whose norm involves all second order derivatives.

However we prove that the standard Hilbertian abstract setting used for solving the elastic problem does not need to be changed. Instead one has to change the definition of the Energy spaces which correspond to the various imposed boundary conditions: they must be regarded as subsets of the Anisotropic Sobolev space whose norm is defined by involving only the derivatives appearing in the considered deformation energy. The abstraction effort due to Nikol'skii (and to Besov and others) which lead him to introduce a wider class of Sobolev spaces was initially motivated only by the need of developing a mathematical theory based on the minimum possible necessary assumptions: Anisotropic Sobolev Spaces include functions which do not posses all higher order weak derivatives.

The abstract tool which he developed allowed us to frame rather naturally the numerical and mathematical problems concerning the equilibrium of linear pantographic sheets. Discussion of the finite element method developed for anisotropic Sobolev's spaces is given in [26].

We are also confident that the same tools will allow us to study non-linear deformations problems. Finally we want to stress once more that the study of apparent pathologies can be a fruitful source of understanding in both mathematics and in the mathematical modelling of physical phenomena.

Acknowledgement V.A.E. acknowledges the financial support by the Russian Science Foundation under grant "Methods of microstructural nonlinear analysis, wave dynamics and mechanics of composites for research and design of modern metamaterials and elements of structures made on its base" (No. 15-19-10008).

Open Access This article is distributed under the terms of the Creative Commons Attribution 4.0 International License (http://creativecommons.org/licenses/by/4.0/), which permits unrestricted use, distribution, and reproduction in any medium, provided you give appropriate credit to the original author(s) and the source, provide a link to the Creative Commons license, and indicate if changes were made.

\section{Appendix: Anisotropic Lebesgue and Sobolev Functional Spaces}

In the paper we used the classic and anisotropic Lebesgue and Sobolev functional spaces. For sake of self consistency, we present here some necessary information on this topic. In plane elasticity and other problems of mechanics functional spaces such as Lebesgue space $L_{2}(\omega)$ and Sobolev's spaces $W_{2}^{1}(\omega)$ and $W_{2}^{2}(\omega)$ are widely used [16, 28, 41, 42]. The norms in these spaces are defined as follows:

$$
\begin{gathered}
\|f\|_{L_{2}}=\left(\iint_{\omega} f^{2} d \omega\right)^{\frac{1}{2}}, \\
\|f\|_{W_{2}^{1}}=\left(\|f\|_{L_{2}}^{2}+\left\|f_{, 1}\right\|_{L_{2}}^{2}+\left\|f_{, 2}\right\|_{L_{2}}^{2}\right)^{\frac{1}{2}}, \\
\|f\|_{W_{2}^{2}}=\left(\|f\|_{L_{2}}^{2}+\left\|f_{, 1}\right\|_{L_{2}}^{2}+\left\|f_{, 2}\right\|_{L_{2}}^{2}+\left\|f_{, 11}\right\|_{L_{2}}^{2}+2\left\|f_{, 12}\right\|_{L_{2}}^{2}+\left\|f_{, 22}\right\|_{L_{2}}^{2}\right)^{\frac{1}{2}},
\end{gathered}
$$

where $f=f\left(x_{1}, x_{2}\right)$ is a function defined in an open set in the two-dimensional Euclidean space, $\omega \subset \mathbb{R}^{2}$, the boundary of $\omega$ is assumed to be smooth enough. Various useful requirements to the boundary of $\omega$ are discussed in $[1,11]$. The Greek indices take values 1,2 . $L_{2}(\omega), W_{2}^{1}(\omega)$ and $W_{2}^{2}(\omega)$ are examples of a separable Hilbert space [1]. 
In this paper we use the various imbedding theorems for Sobolev's spaces, therefore let us recall the general definition of an imbedding. We say that the normed space $E$ is imbedded in the normed space $H$, and we write $E \rightarrow H$ to denote this imbedding, if (i) $E$ is a vector subspace of $H$, and (ii) there exists a constant $C$ such that $\|u\|_{H} \leq C\|u\|_{E} \forall u \in E$. For imbedding theorems in Sobolev's spaces we refer to [1, 41, 42].

In addition to classic Lebesgue and Sobolev's space we introduce the anisotropic Lebesgue and Sobolev spaces. Here we are restricting ourselves to functions defined on a set of $\mathbb{R}^{2}$. Let $\mathbf{p}=\left(p_{1}, p_{2}\right)$ be a multiindex, where $1<p_{\alpha}<\infty$. Then the norm in the anisotropic Lebesgue space $L_{\mathbf{p}}$ is defined as

$$
\|f\|_{L_{\mathbf{p}}}=\left(\left(\int f\left(x_{1}, x_{2}\right)^{p_{1}} d x_{1}\right)^{p_{2} / p_{1}} d x_{2}\right)^{1 / p_{2}} .
$$

If $p_{1}=p_{2}=p$ we use standard notation $L_{\mathbf{p}}=L_{p} \cdot\|\cdot\|_{L_{\mathbf{p}}}$ is called the mixed norm [1].

An anisotropic Sobolev space consists of functions having different differential properties in different coordinate directions, so such functions have generalized derivatives of different order and, in general, different $L_{p}$ in coordinate directions $x_{1}$ and $x_{2}$. The theory of the anisotropic Sobolev spaces including imbedding theorems, relations with other Sobolev's spaces and analysis of the coercivity of differential operators is presented in [9, $10,47]$, see also $[11,60]$. We introduce the multiindex $\ell=\left(l_{1}, l_{2}\right)$ where $l_{\alpha}$ are natural numbers and the norm

$$
\|f\|_{W_{\mathbf{p}}^{\ell}}=\|f\|_{L_{\mathbf{p}}}+\sum_{\alpha=1}^{2}\left\|\partial_{\alpha}^{l_{\alpha}} f\right\|_{L_{\mathbf{p}}} .
$$

So, the set of functions defined on $\omega$ and having generalized derivatives such that the introduced norm is finite, is called the anisotropic Sobolev space $W_{\mathbf{p}}^{\ell}(\omega)$. Obviously, when $l_{1}=l_{2}=l$ and $p_{1}=p_{2}=p$ we have the classical Sobolev space $W_{p}^{l}$. The anisotropic Sobolev's space $W_{\mathbf{p}}^{\ell}$ is a separable Banach space whereas $W_{2}^{\ell}$ is a Hilbert space.

We also introduce the anisotropic Sobolev space $\stackrel{\circ}{W} \underset{\mathbf{p}}{\ell}$ as the closure of $C_{0}^{2}(\omega)\left(\right.$ or $\left.C_{0}^{\infty}(\omega)\right)$ functions in norm (35).

For our purposes we consider two specific anisotropic Sobolev spaces $W_{2}^{(1,2)}$ and $W_{2}^{(2,1)}$ with norms

$$
\begin{aligned}
& \|f\|_{W_{2}^{(1,2)}}=\|f\|_{L_{2}}+\left\|f_{, 1}\right\|_{L_{2}}+\left\|f_{, 22}\right\|_{L_{2}}, \\
& \|f\|_{W_{2}^{(2,1)}}=\|f\|_{L_{2}}+\left\|f_{, 11}\right\|_{L_{2}}+\left\|f_{, 2}\right\|_{L_{2}} .
\end{aligned}
$$

With certain assumptions on the regularity of $\omega$ for these spaces, there are the following imbedding theorems $[11,47]$ :

$$
W_{2}^{\ell}(\omega) \rightarrow W_{2}^{1}(\omega), \quad W_{2}^{\ell}(\omega) \rightarrow C(\omega), \quad \ell=\{(1,2),(2,1)\} .
$$

Evidently, any function $f \in W_{2}^{2}(\omega)$ belongs to $W_{2}^{\ell}(\omega)$ with $\ell=\{(1,2),(2,1)\}$, but not all elements of $W_{2}^{\ell}(\omega)$ belong to $W_{2}^{2}(\omega)$. For more details on imbeddings in anisotropic Sobolev's spaces and their further generalizations including results on traces of functions we refer to [9-11, 47, 48, 60]. 


\section{References}

1. Adams, R.A., Fournier, J.J.F.: Sobolev Spaces, 2nd edn. Pure and Applied Mathematics, vol. 140. Academic Press, Amsterdam (2003)

2. Agmon, S., Douglis, A., Nirenberg, L.: Estimates near the boundary for solutions of elliptic partial differential equations satisfying general boundary conditions, I. Commun. Pure Appl. Math. 12(4), 623727 (1959)

3. Agmon, S., Douglis, A., Nirenberg, L.: Estimates near the boundary for solutions of elliptic partial differential equations satisfying general boundary conditions, II. Commun. Pure Appl. Math. 17(1), 3592 (1964)

4. Agranovich, M.: Elliptic boundary problems. In: Agranovich, M., Egorov, Y., Shubin, M. (eds.) Partial Differential Equations IX: Elliptic Boundary Problems. Encyclopaedia of Mathematical Sciences, vol. 79, pp. 1-144. Springer, Berlin (1997)

5. Auffray, N., dell'Isola, F., Eremeyev, V.A., Madeo, A., Rosi, G.: Analytical continuum mechanics à la Hamilton-Piola least action principle for second gradient continua and capillary fluids. Math. Mech. Solids 20(4), 375-417 (2015)

6. Barozzi, G.C.: Un problema al contorno non omogeneo in un dominio angoloso per equazioni fortemente quasi-ellittiche in due variabili (I). Rend. Semin. Mat. Univ. Padova 44, 27-63 (1970)

7. Barozzi, G.C.: Un problema al contorno non omogeneo in un dominio angoloso per equazioni fortemente quasi-ellittiche in due variabili (II). Rend. Semin. Mat. Univ. Padova 44, 319-337 (1970)

8. Battista, A., Cardillo, C., Vescovo, D.D., Rizzi, N.L., Turco, E.: Frequency shifts induced by large deformations in planar pantographic continua. Int. J. Nanomech. Sci. Technol. 6(2), 161-178 (2015)

9. Besov, O.V., II'in, V.P., Nikol'skii, S.M.: Integral Representations of Functions and Imbedding Theorems, vol. 1 Wiley, New York (1978)

10. Besov, O.V., II'in, V.P., Nikol'skii, S.M.: Integral Representations of Functions and Imbedding Theorems, vol. 2. Wiley, New York (1979)

11. Besov, O.V., II'in, V.P., Nikol'skii, S.M.: Integral Representations of Functions and Imbedding Theorems. Nauka, Moscow (1996) (in Russian)

12. Boutin, C., dell'Isola, F., Giorgio, I., Placidi, L.: Linear pantographic sheets: asymptotic micro-macro models identification. Math. Mech. Complex Syst. 5(2), 127-162 (2017)

13. Cavallucci, A.: Sulle proprietà differenziali delle soluzioni delle equazioni quasi-ellittiche. Ann. Mat. Pura Appl. 67(1), 143-167 (1965)

14. Challamel, N., Kocsis, A., Wang, C.M.: Higher-order gradient elasticity models applied to geometrically nonlinear discrete systems. Theor. Appl. Mech. 42(4), 223-248 (2015)

15. Chambon, R., Moullet, J.C.: Uniqueness studies in boundary value problems involving some second gradient models. Comput. Methods Appl. Mech. Eng. 193(27), 2771-2796 (2004)

16. Ciarlet, P.G.: Mathematical Elasticity, vol. I: Three-Dimensional Elasticity. North-Holland, Amsterdam (1988)

17. Cordero, N.M., Forest, S., Busso, E.P.: Second strain gradient elasticity of nano-objects. J. Mech. Phys. Solids (2016). doi:10.1016/j.jmps.2015.07.012

18. Del Vescovo, D., Giorgio, I.: Dynamic problems for metamaterials: review of existing models and ideas for further research. Int. J. Eng. Sci. 80, 153-172 (2014)

19. dell'Isola, F., Andreaus, U., Placidi, L.: At the origins and in the vanguard of peridynamics, non-local and higher-gradient continuum mechanics: an underestimated and still topical contribution of Gabrio Piola. Math. Mech. Solids 20(8), 887-928 (2015)

20. dell'Isola, F., Della Corte, A., Esposito, R., Russo, L.: Some cases of unrecognized transmission of scientific knowledge: from antiquity to Gabrio Piola's peridynamics and generalized continuum theories. In: Generalized Continua as Models for Classical and Advanced Materials, pp. 77-128. Springer, New York (2016)

21. dell'Isola, F., Della Corte, A., Giorgio, I.: Higher-gradient continua: the legacy of Piola, Mindlin, Sedov and Toupin and some future research perspectives. Math. Mech. Solids 22, 852-872 (2016)

22. dell'Isola, F., Giorgio, I., Pawlikowski, M., Rizzi, N.: Large deformations of planar extensible beams and pantographic lattices: heuristic homogenisation, experimental and numerical examples of equilibrium. Proc. R. Soc. Lond. Ser. A 472(2185), 20150 (2016)

23. dell'Isola, F., Porfiri, M., Vidoli, S.: Piezo-electromechanical (PEM) structures: passive vibration control using distributed piezoelectric transducers. C. R., Méc. 331(1), 69-76 (2003)

24. dell'Isola, F., Seppecher, P., Madeo, A.: How contact interactions may depend on the shape of Cauchy cuts in Nth gradient continua: approach "á la d'Alember”. Z. Angew. Math. Phys. 63(6), 1119-1141 (2012)

25. Dyson, F.: Characterizing irregularity. Science 200(4342), 677-678 (1978) 
26. Eastham, J.F., Peterson, J.S.: The finite element method in anisotropic Sobolev spaces. Comput. Math. Appl. 47(10), 1775-1786 (2004)

27. Egorov, Y.V., Shubin, M.A.: Foundations of the Classical Theory of Partial Differential Equations, 1st edn. Encyclopaedia of Mathematical Sciences, vol. 30. Springer, Berlin (1998)

28. Eremeyev, V.A., Lebedev, L.P.: Existence of weak solutions in elasticity. Math. Mech. Solids 18(2), 204-217 (2013)

29. Eugster, S.R., dell'Isola, F.: Exegesis of the introduction and Sect. I from "Fundamentals of the Mechanics of Continua" by E. Hellinger. Z. Angew. Math. Mech. 97(4), 477-506 (2017)

30. Evans, L.C.: Partial Differential Equations, 2nd edn. Graduate Series in Mathematics, vol. 19. Am. Math. Soc., Providence (2010)

31. Fichera, G.: Linear Elliptic Differential Systems and Eigenvalue Problems. Lecture Notes in Mathematics, vol. 8. Springer, Berlin (1965)

32. Fichera, G.: Existence theorems in elasticity. In: Flügge, S. (ed.) Handbuch der Physik, vol. VIa/2, pp. 347-389. Springer, Berlin (1972)

33. Germain, P.: La méthode des puissances virtuelles en mécanique des milieux continus. Première partie: théorie du second gradient. J. Méc. 12, 236-274 (1973)

34. Germain, P.: The method of virtual power in continuum mechanics, part 2: microstructure. SIAM J. Appl. Math. 25(3), 556-575 (1973)

35. Giorgio, I., Galantucci, L., Della Corte, A., Del Vescovo, D.: Piezo-electromechanical smart materials with distributed arrays of piezoelectric transducers: current and upcoming applications. Int. J. Appl. Electromagn. Mech. 47(4), 1051-1084 (2015)

36. Healey, T.J., Krömer, S.: Injective weak solutions in second-gradient nonlinear elasticity. ESAIM Control Optim. Calc. Var. 15(4), 863-871 (2009)

37. Hörmander, L.: The Analysis of Linear Partial Differential Operators, II: Differential Operators with Constant Coefficients. A Series of Comprehensive Studies in Mathematics, vol. 257. Springer, Berlin (1983)

38. Kline, M.: Mathematical Thought from Ancient to Modern Times: vols. 1, 2,3, vol. 3. Oxford Univ. Press, Oxford (1990)

39. Kuhn, T.S.: The Structure of Scientific Revolutions, 3rd edn. Univ. of Chicago Press, Chicago (1996)

40. Kuznetsov, E.N.: Underconstrained Structural Systems. Mechanical Engineering. Springer, New York (1991)

41. Lebedev, L.P., Vorovich, I.I.: Functional Analysis in Mechanics. Springer, New York (2003)

42. Lebedev, L.P., Vorovich, I.I., Gladwell, G.M.L.: Functional Analysis. Applications in Mechanics and Inverse Problems. Solid Mechanics and Its Applications, vol. 100, 2nd edn. Kluwer, New York (2002)

43. Lions, J.L., Magenes, E.: Non-Homogeneous Boundary Value Problems and Applications, vol. 1. Springer, Berlin (1972)

44. Mareno, A., Healey, T.J.: Global continuation in second-gradient nonlinear elasticity. SIAM J. Math. Anal. 38(1), 103-115 (2006)

45. Matsuzawa, T.: On quasi-elliptic boundary problems. Trans. Am. Math. Soc. 133(1), 241-265 (1968)

46. Mindlin, R.D., Eshel, N.N.: On first strain-gradient theories in linear elasticity. Int. J. Solids Struct. 4(1), 109-124 (1968)

47. Nikol'skii, S.M.: On imbedding, continuation and approximation theorems for differentiable functions of several variables. Russ. Math. Surv. 16(5), 55 (1961)

48. Ohno, M., Shizuta, Y., Yanagisawa, T.: The trace theorem on anisotropic Sobolev spaces. Tohoku Math. J. 46(3), 393-401 (1994)

49. Palamodov, V.P.: Systems of linear differential equations. In: Gamkrelidze, R.V. (ed.) Mathematical Analysis. Progress in Mathematics, pp. 1-35. Springer, Boston (1971)

50. Placidi, L., Andreaus, U., Della Corte, A., Lekszycki, T.: Gedanken experiments for the determination of two-dimensional linear second gradient elasticity coefficients. Z. Angew. Math. Phys. 66(6), 3699-3725 (2015)

51. Placidi, L., Andreaus, U., Giorgio, I.: Identification of two-dimensional pantographic structure via a linear D4 orthotropic second gradient elastic model. J. Eng. Math. 103(1), 1-21 (2017)

52. Placidi, L., Barchiesi, E., Turco, E., Rizzi, N.L.: A review on 2D models for the description of pantographic fabrics. Z. Angew. Math. Phys. 67(5), 121 (2016)

53. Placidi, L., Greco, L., Bucci, S., Turco, E., Rizzi, N.L.: A second gradient formulation for a 2D fabric sheet with inextensible fibres. Z. Angew. Math. Phys. 67(5), 114 (2016)

54. Reda, H., Rahali, Y., Ganghoffer, J.F., Lakiss, H.: Wave propagation in 3D viscoelastic auxetic and textile materials by homogenized continuum micropolar models. Compos. Struct. 141, 328-345 (2016)

55. Rodino, L., Boggiatto, P.: Partial differential equations of multi-quasi-elliptic type. Ann. Univ. Ferrara 45(1), 275-291 (1999) 
56. Scerrato, D., Giorgio, I., Rizzi, N.L.: Three-dimensional instabilities of pantographic sheets with parabolic lattices: numerical investigations. Z. Angew. Math. Phys. 67(3), 1-19 (2016)

57. Sedov, L.I.: Mathematical methods for constructing new models of continuous media. Russ. Math. Surv. 20(5), 123 (1965)

58. Stillwell, J.: Exceptional objects. Am. Math. Mon. 105(9), 850-858 (1998)

59. Toupin, R.A.: Theories of elasticity with couple-stress. Arch. Ration. Mech. Anal. 17(2), 85-112 (1964)

60. Triebel, H.: Theory of Function Spaces III. Monographs in Mathematics, vol. 100. Birkhäuser, Basel (2006)

61. Trinh, D.K., Janicke, R., Auffray, N., Diebels, S., Forest, S.: Evaluation of generalized continuum substitution models for heterogeneous materials. Int. J. Multiscale Comput. Eng. 10(6), 527-549 (2012)

62. Turco, E., Barcz, K., Pawlikowski, M., Rizzi, N.L.: Non-standard coupled extensional and bending bias tests for planar pantographic lattices, part I: numerical simulations. Z. Angew. Math. Phys. 67(5), 122 (2016)

63. Turco, E., Barcz, K., Rizzi, N.L.: Non-standard coupled extensional and bending bias tests for planar pantographic lattices, part II: comparison with experimental evidence. Z. Angew. Math. Phys. 67(123), $1-16(2016)$

64. Turco, E., dell'Isola, F., Rizzi, N.L., Grygoruk, R., Müller, W.H., Liebold, C.: Fiber rupture in sheared planar pantographic sheets: numerical and experimental evidence. Mech. Res. Commun. 76, 86-90 (2016)

65. Turco, E., Golaszewski, M., Cazzani, A., Rizzi, N.L.: Large deformations induced in planar pantographic sheets by loads applied on fibers: experimental validation of a discrete Lagrangian model. Mech. Res. Commun. 76, 51-56 (2016)

66. Turco, E., Rizzi, N.L.: Pantographic structures presenting statistically distributed defects: numerical investigations of the effects on deformation fields. Mech. Res. Commun. 77, 65-69 (2016)

67. Turro, N.J.: Paradigms lost and paradigms found: examples of science extraordinary and science pathological and how to tell the difference. Angew. Chem., Int. Ed. Engl. 39(13), 2255-2259 (2000)

68. Volevich, L.R.: Solubility of boundary value problems for general elliptic systems. Sb. Math. 68(110), 373-416 (1965). (in Russian) 